\title{
VASCULARIZED BONE GRAFTING FOR THE TREATMENT OF SCAPHOID NON-UNION: CASE REPORT
}

\author{
Florin GROSEANU ${ }^{1,2} \otimes$, Stefan CUCULICI ${ }^{1}$, Radu VISAN ${ }^{1,2}$, Bogdan SOCEA ${ }^{2,3}$, \\ Stefan CRISTEA ${ }^{1,2}$
}

${ }^{1}$ Clinical Emergency Hospital "St. Pantelimon“, Department of Orthopedics, Bucharest, Romania

${ }^{2}$ University of Medicine and Pharmacy "Carol Davila", Bucharest, Romania

${ }^{3}$ Clinical Emergency Hospital "St. Pantelimon", Department of General Surgery, Bucharest, Romania

Received 19 Mar 2019, Accepted 06 May 2019

https://doi.org/10.31688/ABMU.2019.54.2.26

\begin{abstract}
Introduction. Scaphoid non-union associated with avascular proximal pole necrosis represent a really challenging problem for orthopedic surgeons and its treatment is still in debate.
\end{abstract}

Case presentation. We present the case of a 33-year-old patient, with scaphoid non-union, who was treated according to Zeidenberg's technique using a vascularized radial bone graft. The primary objectives of treatment were to obtain union of the scaphoid, to improve the functional recovery of the wrist and to allow professional reimplantation of the patient. We were able to obtain a functional graft and after 9 months the results were very good.

Conclusions. Vascularized bone grafts represent the optimal method of treatment of scaphoid non-union associated to avascular proximal pole. It is mandatory to be associated to rigid osteosynthesis such as Herbert compression screw. The technique of Zeidenberg offers good results in these cases, but it should not be performed without magnifying lens and tourniquet uses.

\section{Résumé}

Greffe vascularisée radiale pour le traitement de la pseudarthrose de l'os scaphoïde: présentation de cas

Introduction. La pseudarthrose du scaphoïde associée à la nécrose du pôle proximal représente un problème thérapeutique pour les chirurgiens orthopèdes et son traitement est encore discutable.

Présentation du cas. On présente le cas d'un patient de 33 ans avec pseudarthrose du scaphoïde qui a été traité selon la technique de Zeidenberg en utilisant une greffe osseuse vascularisée radiale. Le but du traitement est d'obtenir la consolidation du scaphoïde, la récupération fonctionnelle et l'insertion professionnelle du patient. Nous avons réussi à obtenir une greffe fonctionnelle et les résultats à 9 mois étaient parfaits.

Conclusions. Les greffes osseuses vascularisées représentent la méthode optimale de traitement des non-unions scaphoïdes associées au pôle proximal avasculaire. Il est obligatoire d'être associé à une ostéosynthèse rigide telle que la vis de compression de Herbert. La technique de Zeidenberg offre de bons résultats 
Keywords: scaphoid non-union, conventional grafts, vascularized bone graft, Zeidenberg's technique.

\section{Abbreviations list:}

SNAC = scaphoid non-union advanced collapse; MRI = magnetic resonance imaging; $1,2 \mathrm{ICSRA}=1,2$ intercompartment supra retinacular artery.

\section{INTRODUCTION}

Described by Barton" as an "awkward but important little bone", the scaphoid is the most commonly fractured carpal bone, second in frequency to distal radius fractures. Due to its unique source of vascularization $^{2}$ via the radial artery, $70 \%-80 \%$ of its intra-osseous vascularity protruding distally and dorsally into the bone, non-union of scaphoid fractures is the most common complication associated with this pathology. Untreated, non-union progresses to scaphoid non-union advanced collapse (SNAC) which implies arthritic changes of the radio-scaphoid and midcarpal joints and finally pan-carpal arthritis ${ }^{3}$. Mack $^{4}$ found out $97 \%$ patients with arthritic changes at 5 years after established non-union and that these changes are proportionate to duration of evolution of this disease.

Matti-Russe technique ${ }^{5}$ for scaphoid non-union treatment has been the standard protocol for the last 50 years, but the results are not always good. In the last two decades attention was focused on vascularized bone grafting, especially for proximal pole or waist non-union.

\section{Case presentation}

A 33-year-old patient presented in our clinic for pain and limited mobility of the non-dominant left dans ces cas, mais elle ne devrait pas être réalisée sans grossissements de loupes et utilisation de garrot.

Mots-clés: pseudarthrose du scaphoïde, greffe conventionnelle, greffe radiale vascularisée, technique de Zeidenberg.

wrist. He reported a fall on his hand 7 months before; at that time, he was diagnosed with fracture of the scaphoid and received orthopedic treatment consisting in ante brachial palmar digital casting for 6 weeks, after which he was referred to a rehabilitation center. Despite tenuous and prolonged kineto-therapy protocol, the symptomatology didn't regress.

When he presented at our department, the clinical exam revealed pain on the snuffbox and over the dorsum of the wrist, reduced extension and marked reduced cubital inclination of the wrist, an almost normal flexion and radial inclination. The X-ray showed a scaphoid proximal pole non-union. The MRI showed synovial pseudarthrosis, with early signs of avascular necrosis of the proximal pole, but with normal carpal alignment (Figure 1).

We admitted the patient for surgery and, due to the location of non-union and the presence of osteonecrosis of the proximal pole, we considered that a vascularized bone grafting according to Zaidenberg's technique $^{6}$ (Figure 2), using the 1,2 intercompartment supra retinacular artery (1,2 ICSRA) graft, was the optimal method of treatment. Intervention was done using tourniquet and wens magnification $4 x$. We used a longitudinal dorsal radial incision paralleling extensor pollicis longus tendon and the 1,2 ICSRA was found between the $1^{\text {st }}$ and $2^{\text {nd }}$ compartments of the extensor retinaculum. The center of the

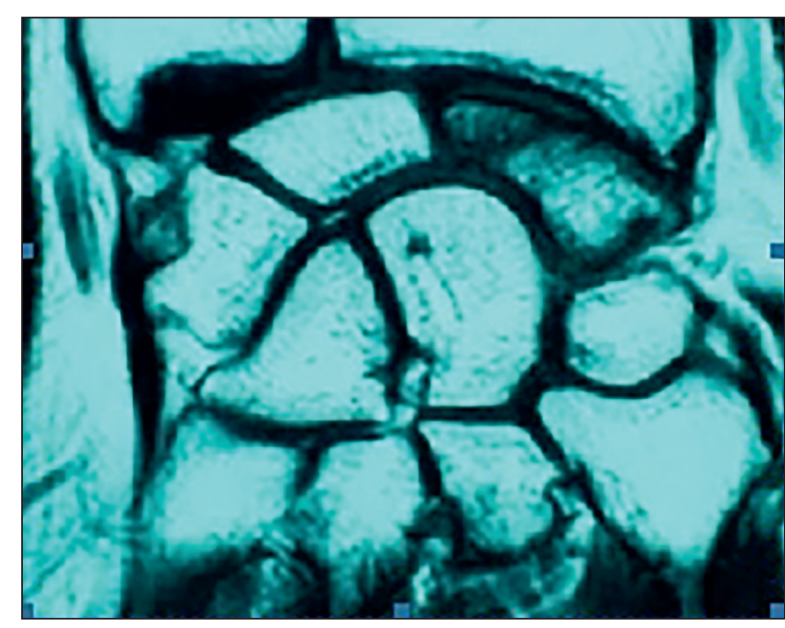

Fig.1. MRI showing avascular proximal pole

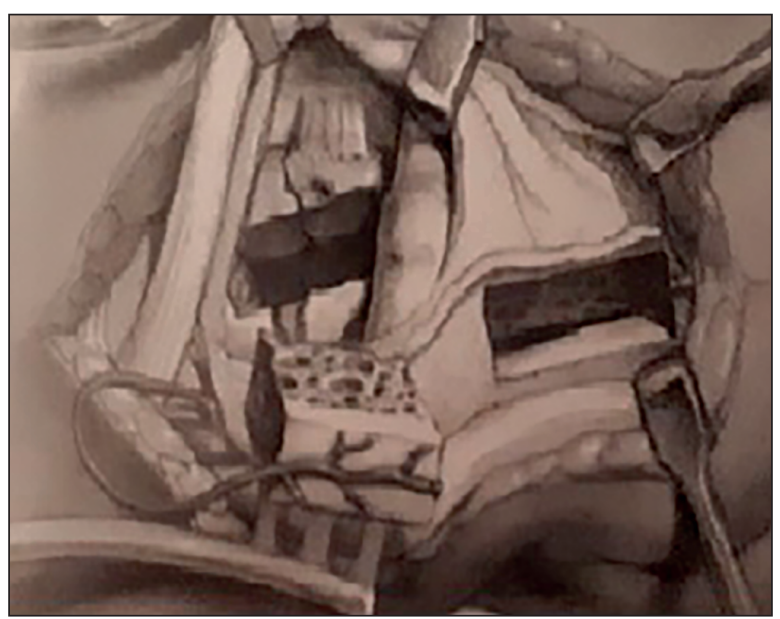

Fig.2. 1,2 ICSRA radial graft 


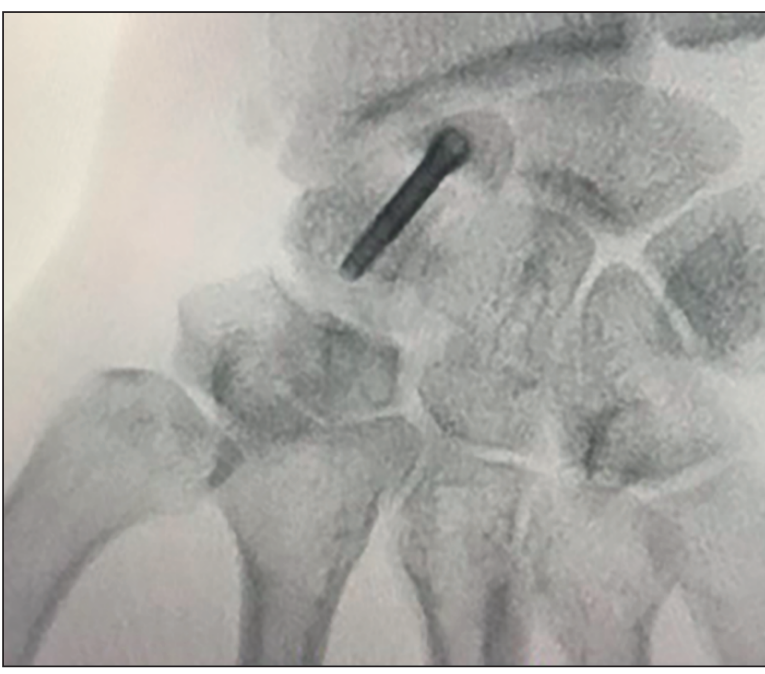

Fig. 3. Intraoperative X-ray

graft was $1.5 \mathrm{~cm}$ proximal to the radio-scaphoid joint, to include the nutrient vessels that enter into the radial styloid in that area. We didn't perform partial radial styloidectomy. After the site of non-union was scraped of the fibrous tissue, the graft was gently put into place. Internal fixation was done using a Herbert compression screw placed to avoid dislodging of the graft (Figure 3).

Postoperatively, we used a wrist orthosis for 8 weeks, allowing immediate finger movements. At 8 weeks, the orthosis was removed and the patient begun wrist movements, but strengthening program was permitted after another 4 weeks. The X-ray done at 6 months (Figure 4) showed union of the scaphoid and the clinical exam revealed pain-free and complete flexion, radial inclination, cubital inclination and prone supination of the wrist and 15\% lack of extension. At 9 months, the extension was complete.

\section{Discussion}

Scaphoid non-union associated with avascular proximal pole necrosis represents a real challenging problem for orthopedic surgeons and its treatment is still in debate. The Matti-Russe ${ }^{5}$ volar inlay graft has poor results in these cases ${ }^{7}$, because the proximal fragment has no potential for consolidation. Bad results are also seen in cases using conventional $\mathrm{graft}^{8}$ (from the iliac crest, distal radius), especially when compression across non-union site is not achieved, such as in cases using Kirschner wires as osteosynthesis; but even when Herbert screws are used, the rate of non-union is up to $53 \%$.

The technique of proximal pole excision and tendon interposition ${ }^{9}$ (taken from palmaris longus or

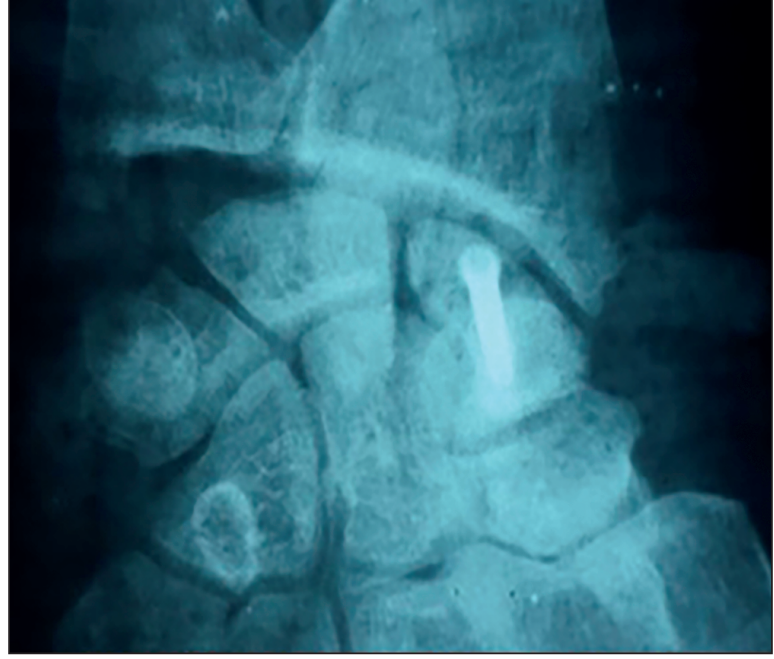

Fig. 4. X-ray at 6 months postoperative

flexor radialis carpi) is not recommended anymore, because it alters the kinematic of the wrist (due to absence of scapho-lunate ligament) and will finally lead to wrist arthrosis.

There are many techniques that use vascularized bone graft: pronator quadratus grafts ${ }^{10}$, proximal second metacarpal metaphysis ${ }^{11}$, palmar and dorsal radial distal metaphysis ${ }^{11-13}$, medial femoral condyle ${ }^{14}$, iliac crest associated to arteriovenous bundle ${ }^{15}$. These techniques provide better union rates compared to conventional grafts, especially for patients with previous failed surgery ${ }^{8}$. The pronator quadratus vascularized graft has the disadvantage of a small pedicle and highly variable in location.

We preferred the technique of Zeidenberg ${ }^{6}$, described for the first time in 1991, because the graft is easily accessible, the arterial pedicle of the graft is long enough to reach the site of non-union and in the literature the results are very good. Zeidenberg ${ }^{6}$ reports successful results in 11 cases $(100 \%$ union rates) and Steinmann ${ }^{16}$ in 15 cases.

The 1,2 ICSRA originates from the radial artery $5 \mathrm{~cm}$ proximal to wrist joint and anastomoses to the radiocarpal arch or radial artery distal at the level of the snuffbox. It gives branches that enter the distal radius approximately $1.5 \mathrm{~cm}$ proximal to radiocarpal joint. We were able to identify this artery and before closure, after tourniquet release, we found out that it was functional. We didn't perform radial styloidectomy, because we didn't have problems of visualization during surgery. At 9 months the graft consolidated and wrist movements were almost normal. The patient will be followed-up annually to see if in time he will develop wrist arthrosis. 


\section{Conclusions}

Vascularized bone grafts represent the optimal method of treatment of scaphoid non-union associated to avascular proximal pole. It is mandatory to be associated to rigid osteosynthesis such as Herbert compression screw. The technique of Zeidenberg offers good results in these cases, but it should not be performed without magnifying lens and tourniquet uses. Dissection must be done carefully because the small arterioles can be damaged very easy and before closure the function of these vessels must be evaluated.

\section{Compliance with Ethics Requirements:}

„The authors declare no conflict of interest regarding this article"

"The authors declare that all the procedures and experiments of this study respect the ethical standards in the Helsinki Declaration of 1975, as revised in 2008(5), as well as the national law. Informed consent was obtained from the patient included in the study"

"No funding for this study"

\section{References}

1. Barton NJ. Apparent and partial non-union of the scaphoid. Journal of Hand Surgery [Br]. 1996;21:496-500.

2. Gelberman RH, Menon J. The vascularity of the scaphoid bone. Journal of Hand Surgery [Am.] 1980;5(5):508-513.

3. Geissler WB, Slade JF. Fractures of the carpal bones. In Green's Operative Hand Surgery, $6^{\text {th }}$ edition, Edit. Elsevier, Vol 1, 2011;18:639-707.

4. Mack GR, Bosse MJ, Gelberman RH, et al. The natural history of scaphoid non-union, Journal of Bone Joint Surgery [Am]. 1984;66(4):504-509.
5. Russe O. Fractures of the carpal navicular. Journal of Bone Joint Surgery [Am]. 1960; 42-A:759-768.

6. Zeidenberg C, Siebert JW, Angrigiani C. A new vascularized bone graft for scaphoid nonunion. Journal of Hand Surgery [Am]. 1991;16(3):474-478.

7. Dacho A, Germann G, Sauerbier M. The reconstruction of scaphoid pseudarthrosis with the operation of Matti-Russe, A retrospective follow-up analysis of 84 patients. Unfallchirurgie. 2004;107(5):388-396.

8. Merrell GA, Wolfe SW, Slade JF III. Treatment of scaphoid non-unions: quantitative meta-analysis of the literature, Journal of Hand Surgery [Am]. 2002;27(4):685-691.

9. Wright PE. Wrist. In Campbell's Operative Orthopaedics, $8^{\text {th }}$ edit, edited by Crenshaw A.H., Mosby, 1992, vol 5, 66:3123-3166.

10. Sheetz KK, Bishop AT, Berger RA. The arterial blood supply of the distal radius and ulna and its potential use in vascularized pedicled bone grafts. Journal of Hand Surgery [Am]. 1995;20(6):902-914.

11. Pierer G, Steffen J, Hoflehner H. The vascular blood supply of the second metacarpal bone: anatomic basis for a new vascularized bone graft in hand surgery. An anatomical study in cadavers. Surgery and radiologic anatomy. 1992;14(2):103-112.

12. Henry M. Collapsed scaphoid non-union with dorsal intercalated segment instability and avascular necrosis treated by vascularized wedge-shaped bone graft and fixation. Journal of Hand Surgery [Br]. 2007;32(2):148-154.

13. Kuhlmann JN, Mimoun M, Boabighi A, Baux S. Vascularized bone graft pedicled on the volar carpal artery for non-union of the scaphoid. Journal of Hand Surgery [Br]. 1987;12(2):203-210.

14. Larson AN, Bishop AT, Shin AY. Free medial femoral condyle bone grafting for scaphoid nonunion with humpback deformity and proximal pole avascular necrosis. Technique of Hand and Upper Extremities Surgery. 2007;11(4):246-258.

15. Fernandez DL, Eggli S. Non-union of the scaphoid: revascularization of the proximal pole with implantation of a vascular bundle and bone-grafting. Journal of Bone Joint Surgery [Am]. 1995;77(6):883-893.

16. Steinmann SP, Bishop AT, Berger RA. Use of the 1,2 intercompartmental supra retinacular artery as a vascularized pedicle bone graft for difficult scaphoid nonunion. Journal of Hand Surgery [Am]. 2002;27(3):391-401. 